\title{
Computed Tomography: from Photon Statistics to Modern Cone-Beam CT
}

Thorsten M. Buzug

Berlin, Germany: Springer, 2008, \$109

This book provides an overview of x-ray technology, describes the historic developmental milestones of modern CT systems, and gives comprehensive insight into reconstruction methods. Its intended audience is graduate students in biomedical engineering, electrical engineering, and medical physics.

This book is different from many books on x-ray physics and $\mathrm{CT}$ in the way the reconstruction methods are treated. There is an abundance of equations to describe the reconstruction methods for 2-dimensional CT and cone-beam CT. Scattered among many different journals are descriptions of the various CT reconstruction methods: from 2-dimensional parallel-beam, fanbeam, helical scan trajectory, and cardiac CT to 3-dimensional cone-beam. The author did an excellent job of bringing these descriptions together into a single book.

The book consists of an introductory chapter followed by chapters on the fundamentals of $\mathrm{x}$-ray physics, the milestones of CT, the fundamentals of signal processing, 2-dimensional Fourier-based reconstruction, algebraic and statistical reconstruction, technical implementations, 3-dimensional Fourier-based reconstruction, image quality and artifacts, practical aspects of CT, and dose.

One drawback of this book is the 3-dimensional images. The sagittal and coronal images in Figures 1.8, 9.26, and 9.28 are not representative of images from modern CT scanners.

I strongly recommend this book for students, scientists, and physicists who are interested in reconstruction theory and algorithms. For people who are not as interested in the equations, chapters $1-3$ and 9-10 will enrich their knowledge of CT. 